\title{
Predictors for outcome of failure of balloon dilatation in patients with achalasia
}

\author{
J Alderliesten, ${ }^{1} \mathrm{~J}$ M Conchillo, ${ }^{1}$ I Leeuwenburgh, ${ }^{1}$ E W Steyerberg, ${ }^{2}$ E J Kuipers ${ }^{1,3}$
}

'Department of

Gastroenterology and

Hepatology, Erasmus MC University Medical Centre Rotterdam, The Netherlands ${ }^{2}$ Public Health, Erasmus MC University Medical Centre Rotterdam, The Netherlands ${ }^{3}$ Internal Medicine, Erasmus MC University Medical Centre Rotterdam, The Netherlands

\section{Correspondence to}

Dr J Alderliesten, Department of Gastroenterology and

Hepatology, Erasmus University Medical Centre, PO Box 2040,

Rotterdam $3000 \mathrm{CA}$, The

Netherlands:

jalderliesten@asz.n

Revised 12 October 2010 Accepted 13 October 2010 Published Online First 10 November 2010
ABSTRACT

Background Pneumatic balloon dilatation (PD) is a regular treatment modality for achalasia. The reported success rates of PD vary. Recurrent symptoms often require repeated $P D$ or surgery.

Objective To identify predicting factors for symptom recurrence requiring repeated treatment.

Methods Between 1974 and 2006, 336 patients were treated with PD and included in this longitudinal cohort study. The median follow-up was 129 months (range 1-378). Recurrence of achalasia was defined as symptom recurrence in combination with increased lower oesophageal sphincter (LOS) pressure on manometry, requiring repeated treatment. Patient characteristics, results of timed barium oesophagram and manometry as well as baseline PD characteristics were evaluated as predictors of disease recurrence with Kaplan-Meier curves and Cox regression analysis.

Results 111 patients had symptom recurrence requiring repeated treatment. Symptoms recurred after a mean follow-up of 51 months (range 1-348). High recurrence percentages were found in patients younger than 21 years in whom the 5 and 10-year risks of recurrence were $64 \%$ and $72 \%$, respectively. These risks were respectively $28 \%$ and $36 \%$ in patients with classic achalasia, respectively $48 \%$ and $60 \%$ in patients without complete obliteration of the balloon's waist during PD and respectively $25 \%$ and $33 \%$ in patients with a LOS pressure greater than $10 \mathrm{~mm} \mathrm{Hg}$ at 3 months post-dilatation. These four predictors remained statistically significant in a multivariable Cox analysis. Conclusion Although PD is an effective primary treatment in patients with primary achalasia, patients are at risk of recurrent disease, with this risk increasing during long-term follow-up. Young age at presentation, classic achalasia, high LOS pressure 3 months after PD and incomplete obliteration of the balloon's waist during $\mathrm{PD}$ are the most important predicting factors for the need for repeated treatment during follow-up. Patients who meet one or more of these characteristics may be considered earlier for alternative treatment, such as surgery.

Achalasia is a rare condition, with an estimated annual incidence of one per 100000 , characterised by a functional obstruction of the oesophagus caused by failed relaxation of the lower oesophageal sphincter (LOS) in combination with absent peristalsis of the distal oesophagus. ${ }^{1}$ The aetiology of achalasia is still unknown. The disease is characterised by a loss of inhibitory nitrinergic neurons in the oesophageal myenteric plexus. Proposed causes include genetic inheritance, neuronal degeneration, viral infection and autoimmune disease. ${ }^{2}$ The characteristic symptoms of patients with achalasia are dysphagia for solids and liquids, regurgitation of

\section{Significance of this study}

What is already known about this subject?

- Several studies have reported success rates of PD between $35 \%$ and $85 \%$.

- The previous identified risk factors for symptom recurrence after $P D$ were mainly young age $(<40$ years), a post-treatment LOS pressure above $10 \mathrm{~mm} \mathrm{Hg}$ and a short period of symptoms before treatment.

- Laparoscopic myotomy combined with an antireflux procedure provides better symptom relief than all endoscopic and other surgical approaches, with a low complication rate.

\section{What are the new findings?}

- After treatment with PD we found a 5-year risk of recurrence of $28 \%$.

- Given the long follow-up period in our study, we were able to show that the rate of recurrence does increase over time.

- Young age at presentation, a manometric pattern of classic achalasia, high LOS pressure 3 months after PD, and incomplete obliteration of the balloon"s waist during PD are the most important predicting factors for the need for repeated treatment during follow-up.

How might it impact on clinical practice in the foreseeable future?

- This implies that a stricter follow-up can be given to patients with the above-mentioned predicting factors, as the chance of recurrence is significantly higher in this subgroup. Using a stricter follow-up, recurrence (defined as symptom recurrence in combination with objective parameters such as a significant increase in LOS pressure) can be diagnosed at an earlier stage.

- Patients who meet one or more of these characteristics may be considered earlier for alternative treatment, such as surgery.

undigested food or saliva, respiratory complaints (nocturnal cough, aspiration), retrosternal pain and weight loss. Therapeutic options aim to improve oesophageal emptying by decreasing LOS pressure. There are several treatment options, either endoscopic (botulinum toxin injection and pneumatic balloon dilatation (PD)) or surgical. ${ }^{3}$ Most gastroenterologists prefer PD as first-line therapy. Nevertheless, the reported success rates of $\mathrm{PD}$ vary widely, ranging in previous studies from $35 \%$ to $85 \%$ within several years of follow-up. ${ }^{4-11}$ These marked differences may be partly explained by online under the BMJ Journals unlocked scheme, see http:// gut.bmi.com/site/about/ unlocked.xhtml 
differences in the length of follow-up. Recurrence of symptoms often requires repeated $\mathrm{PD}$ or further treatment with surgery. The major determinants for PD therapy failure are insufficiently known; such knowledge would be clinically relevant as it may aid in targeted surveillance and early intervention in high-risk groups. Several studies have therefore tried to identify predicting factors of clinical outcome following PD. Some did not find any specific predictor. ${ }^{10}$ Other studies found that advanced age, female gender, a long history of symptoms before diagnosis, high pre-therapeutic LOS pressure, a post-dilatation LOS pressure of less than $10 \mathrm{~mm} \mathrm{Hg}$, limited contrast retention on barium oesophagram post-dilatation and a higher number of repeated baseline PD dilatations were associated with a favourable treatment outcome. ${ }^{5} \quad 6 \quad 8 \quad 11-15$ These studies were often performed with relatively small groups of patients. The aim of this study was therefore to identify predicting factors for symptom recurrence requiring repeated $\mathrm{PD}$ in a large cohort of patients with primary achalasia.

\section{PATIENTS AND METHODS \\ Patients}

From 1974 to 2006, 433 patients (214 men and 219 women), mean age $51.1 \pm 21.6$ years (range $4-92$ ) were diagnosed with primary achalasia in our centre. Their data were prospectively included in a database, and they were treated and followed according to a strict patient management, treatment and follow-up protocol, which remained unchanged throughout the study period.

\section{Diagnosis}

The diagnosis of achalasia was based on results from gastroscopy, manometry and timed barium oesophagram.

\section{Endoscopy}

Upper gastrointestinal endoscopy using a fibre or video-endoscope (Olympus Europe, Hamburg, Germany) was performed to rule out secondary achalasia.

\section{Oesophageal manometry}

Oesophageal manometry was performed using a low compliance, pneumohydraulic water infusion system (Medical Measure Systems, Enschede, The Netherlands) and a four-channel silicone catheter (Medical Measure Systems). From May 2005 onwards we used an eight-channel water-perfused manometry assembly with an incorporated $6 \mathrm{~cm}$ long reversed-perfused sleeve sensor (Dentsleeve International Ltd, Mississauga, Ontario, Canada). The recording sites were connected to an eight-channel polygraph (Medical Measure Systems). The fourchannel catheter was introduced with all four recording sites in the stomach and then manually pulled back per centimetre with a wet swallow of $5 \mathrm{cc}$ water at each centimetre. The eightchannel catheter was introduced in the stomach and then manually pulled back till the sleeve was positioned at the level of the LOS. Mean resting LOS pressure was calculated as the mean end-expiratory LOS pressure during 10 wet swallows. LOS relaxations were considered to be absent if no change was recorded after swallowing and complete if swallow-induced LOS pressure returned to the basic intragastric level. A pressure fall above basic gastric level was considered as partial relaxation. Peristaltic wave amplitudes were also calculated as mean wave amplitudes during 10 wet swallows. Aperistalsis was diagnosed if all contractions after swallowing were simultaneous. We differentiated two patterns of aperistalsis. Classic achalasia was defined with the presence of non-transmitted or simultaneous contractions with a low amplitude. Vigorous achalasia was diagnosed if there were simultaneous contractions with an amplitude of more than $37 \mathrm{~mm} \mathrm{Hg}$

\section{Timed barium oesophagram}

Timed barium swallow was performed in the standing position after an overnight fast. The patient was asked to drink $200 \mathrm{cc}$ barium solution (or as much as tolerated without regurgitation or aspiration) and recordings of the oesophagus were performed at 0 , 1 and 10 min after the last barium swallow. The appearance of a so-called 'bird's beak' was recorded, and the height and maximal width of the barium column were measured at 1 and $10 \mathrm{~min}$.

\section{Pneumatic dilatation}

From 1974 to 1976, homemade pneumodilators were used. Since then, dilation balloons became commercially available, and PD procedures were performed using a Rigiflex balloon (Boston Scientific, Natick, Massachusetts, USA).

Baseline PD was performed on three consecutive days with balloons of either the same or incremental (30, 35 and $40 \mathrm{~mm}$ ) diameter. We initially used a $40 \mathrm{~mm}$ balloon for all three consecutive dilations. Under the assumption of lower perforation risk, we later adapted this protocol to a graduated approach using balloons with incremental diameters of 30,35 and $40 \mathrm{~mm}$, respectively, inflated like before on successive days. In the case of recurrence, we performed a new series of dilatations on three consecutive days, again with the same protocol as used for initial treatment. After changing our protocol to the graduated approach, all patients with a recurrence were treated with this protocol, irrespective of the earlier balloon diameter protocol used. Under conscious sedation the balloon was positioned fluoroscopically at the gastro-oesophageal junction and inflated to a pressure of $300 \mathrm{~mm} \mathrm{Hg}$ for $1 \mathrm{~min}$. Until mid-2001 patients were hospitalised for $4-5$ days, but later we switched to an outpatient procedure.

We only performed a post-procedural oesophagram using water-soluble contrast on suspicion of a perforation after PD. Dilation of the oesophagus to any extent on a timed barium oesophagram was no exclusion criterion for PD.

\section{Post-dilatation follow-up}

Following PD, all patients had a strict schedule of follow-up at 3 , 12, 24 months and then every 2 years. Surveillance included gastroscopy, manometry and timed barium oesophagram. After 8 years of follow-up surveillance included gastroscopy; further examination was performed on indication. This schedule was consistently followed for all patients from 1974.

Recurrence of achalasia was defined as relapse of symptoms (in particular dysphagia, regurgitation and weight loss) in combination with an increased LOS pressure on manometry in comparison with the previous LOS pressure, requiring further treatment. We did not use a specific quantified symptom instrument to define symptom recurrence. Primary failure of treatment was defined as the persistence or recurrence of symptoms in the first 3 months after PD.

\section{Evaluation of predictors of disease recurrence}

Patient characteristics (age at presentation, gender), the results of timed barium oesophagram, manometry before treatment, PD balloon characteristics (stable vs incremental diameter and complete vs incomplete obliteration of the balloon's waist during PD), as well as the results of repeated manometry 3 months post-treatment were evaluated as predictors of disease recurrence. 
Time to recurrence was calculated from first PD. Kaplan-Meier curves were constructed to estimate the 5 and 10 -year cumulative incidence of recurrence. Cox proportional hazards regression was used to estimate univariable and multivariable HR. Associations between continuous predictors and recurrence were analysed with restricted cubic spline functions on the log hazard scale. ${ }^{16}$ These splines allow for a flexible fit of the non-linear effects of predictors with relatively few degrees of freedom (df). The predictive strength of each predictor in the multivariable model was indicated by a $\chi^{2}$ statistic, based on the difference in $-2 \log$ likelihood of a model with and without the predictor. ${ }^{17}$ Statistical analysis was performed using SPSS (version 12.0), and $\mathrm{R}$ software (version 2.5.1).

\section{RESULTS}

\section{Patient characteristics}

From 1974 to 2006, 433 patients were diagnosed with achalasia based on the above-mentioned criteria. Thirty-seven of them did not undergo PD for various reasons including failure of previous treatment elsewhere, or direct preference for cardiomyotomy. The remaining 396 patients were treated with PD. Sixty (15.2\%) of them were excluded from analysis as they had undergone previous endoscopic or surgical treatment elsewhere. The remaining 336 patients were included for analysis, including 161 men and 175 women, with a mean age at initial presentation of 50 years (range 4-92). In 288 of 336 patients a manometric subclassification of achalasia could be made. There were 222 (77\%) patients with classic achalasia and 66 (23\%) patients with vigorous achalasia. The median follow-up of all patients was 129 months (range 1-378). During follow-up 94 (28\%) patients died and 74 (22\%) patients were lost to follow-up. Two-hundred and twelve patients (63\%) were at baseline treated with the fixed $40 \mathrm{~mm}$ balloon protocol, 63 (19\%) were treated with the incremental balloon protocol, and the remaining 61 patients were treated with another dilatation protocol, fixed $30 \mathrm{~mm}$ balloon, fixed $35 \mathrm{~mm}$ balloon or unknown protocol.

\section{Complications}

Among a total of $985 \mathrm{PD}$ performed in 336 patients, 40 (4.1\%) procedures were associated with one or more complications. The complications are summarised in table 1. In 13 (1.3\%) of the 985 PD, an oesophageal perforation occurred. Two of these patients required surgery; one patient underwent primary repair of the oesophagus and one patient oesophageal resection. An oesophageal stent was temporarily placed in one patient. The other 10 patients underwent conservative treatment with antibiotics and no oral feeding. There was no mortality and all patients had a good clinical outcome and were discharged $17 \pm 7.9$ days after the perforation. Perforation occurred in five $(2.4 \%)$ of the 212 patients who were repeatedly treated with a $40 \mathrm{~mm}$ balloon,

Table 1 Complications related to PD

\begin{tabular}{lll}
\hline Complication & $\begin{array}{l}\text { No of procedures } \\
(\mathbf{n = 9 8 5 )}\end{array}$ & $\begin{array}{l}\text { Percentage } \\
(\%)^{*}\end{array}$ \\
\hline None & 945 & 95.9 \\
Post-procedural & 31 & 3.1 \\
pain & 16 & 1.6 \\
Fever & 13 & 1.3 \\
Perforation & 3 & 0.3 \\
Aspiration & 2 & 0.2 \\
Bleeding & 2 & \\
\hline
\end{tabular}

*Proportions do not add up to $100 \%$ as some patients had more than one complication, such as the combination of post-procedural pain, fever and perforation.

$\mathrm{PD}$, pneumatic balloon dilatation. compared with one $(1.6 \%)$ of the 63 patients who were treated with incremental size balloons.

Six perforations occurred in patients who were treated with a fixed dilatation protocol with a $35 \mathrm{~mm}$ balloon. One perforation occurred in a patient with an unknown dilatation protocol. Perforations occurred in five of 161 (3.1\%) men versus eight of $175(4.6 \%)$ women $(p<0.001)$. They were also more common in patients with complete obliteration of the balloon waist (complete vs incomplete 5.1 vs $1.8 \%, \mathrm{p}<0.001$ ). There were no associations between perforation rates and age, LOS pressure on manometry before $\mathrm{PD}$, or duration of achalasia symptoms before diagnosis.

\section{Overall outcome}

During follow-up, 111 of the 336 patients developed recurrence of achalasia requiring further treatment. These first recurrences occurred at a mean interval of 51 months (range 1-348) after baseline treatment (figure 1). In 12 patients the symptoms persisted or recurred within the first 3 months after PD.

Recurrences were treated with renewed PD in 88 (79\%) of the 111 patients, the remainder received other treatment modalities, in particular cardiomyotomy (9\%) and botulinum toxin injection (3.6\%). The 5-year risk of recurrence was 28\%; the 10 -year risk of recurrence was $34 \%$ (table 2 ).

Forty-six of the 88 patients who had been treated with repeated $\mathrm{PD}$ for disease recurrence developed a second recurrence of achalasia at a mean interval of 70 months (range 13-372) after renewed $\mathrm{PD}$. Second recurrences were treated with renewed PD in 34 (74\%) of the 46 patients. Fifteen of those 34 patients developed a third recurrence of achalasia at a mean interval of 109 months (range 22-242) after renewed PD and were then treated with renewed PD in nine $(60 \%)$ of the 15 patients.

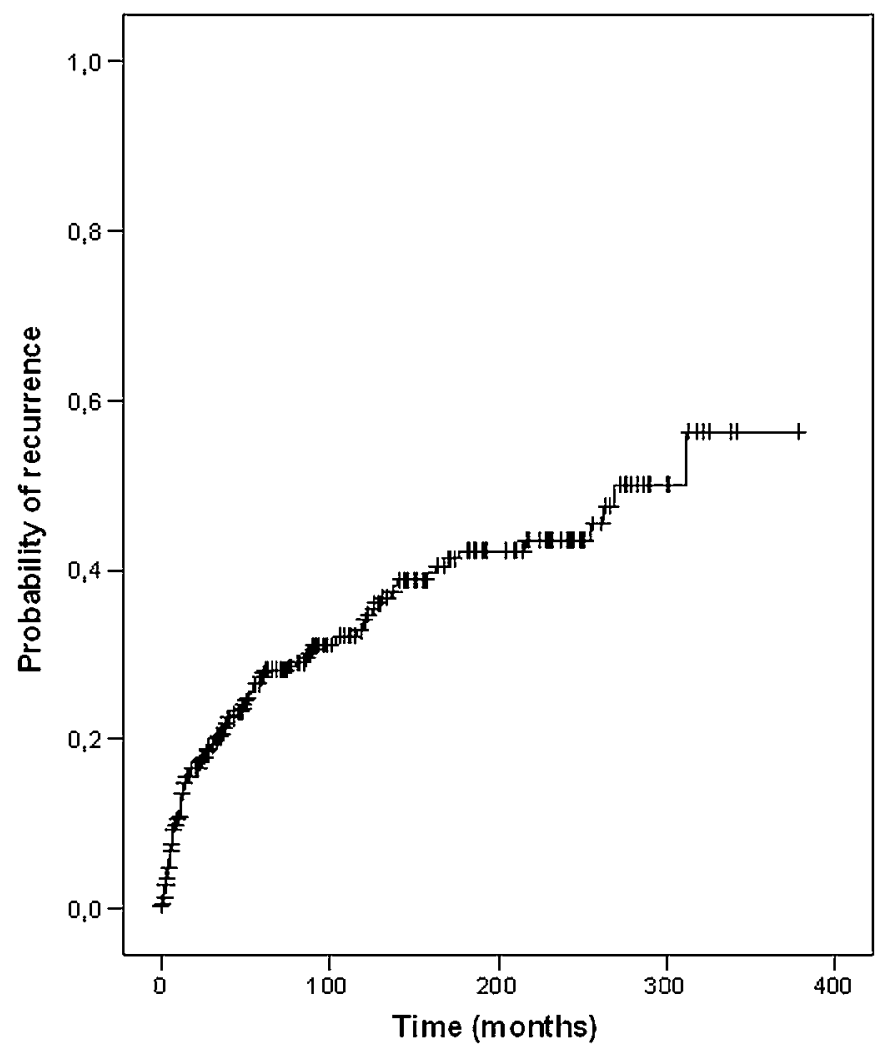

Figure 1 Kaplan-Meier curve for the probability of symptom recurrence after successful primary pneumatic balloon dilatation for achalasia. 
Table 2 Distribution of patient and treatment characteristics in relation to recurrence of achalasia

\begin{tabular}{|c|c|c|c|c|c|c|}
\hline Characteristic & $\mathbf{n}$ & $\begin{array}{l}\text { Recurrence } \\
\text { (n) }\end{array}$ & $\begin{array}{l}\text { 5-Year risk of } \\
\text { recurrence (SE) }\end{array}$ & $\begin{array}{l}10-Y e a r \text { risk of } \\
\text { recurrence (SE) }\end{array}$ & HR (95\% Cl) & p Value \\
\hline \multicolumn{7}{|l|}{ Sex } \\
\hline Male & 161 & 52 & $0.27 \pm 0.04$ & $0.33 \pm 0.04$ & 1.0 & 0.93 \\
\hline Female & 175 & 59 & $0.29 \pm 0.04$ & $0.37 \pm 0.04$ & $1.02(0.7$ to 1.5$)$ & \\
\hline \multicolumn{7}{|l|}{ Age (years) at onset } \\
\hline$<21$ & 31 & 23 & $0.64 \pm 0.9$ & $0.72 \pm 0.09$ & 1.0 & $<0.001$ \\
\hline $21-40$ & 91 & 42 & $0.31 \pm 0.5$ & $0.38 \pm 0.06$ & $0.4(0.3$ to 0.7$)$ & \\
\hline $40-50$ & 46 & 11 & $0.17 \pm 0.6$ & $0.33 \pm 0.10$ & $0.3(0.1$ to 0.5$)$ & \\
\hline$>50$ & 168 & 35 & $0.21 \pm 0.3$ & $0.24 \pm 0.04$ & $0.2(0.1$ to 0.4$)$ & \\
\hline \multicolumn{7}{|l|}{ Type of achalasia } \\
\hline Classic & $222 / 288$ & 79 & $0.28 \pm 0.03$ & $0.36 \pm 0.04$ & $1.8(1.0$ to 3.2$)$ & 0.032 \\
\hline Vigorous & $66 / 288$ & 15 & $0.18 \pm 0.05$ & $0.21 \pm 0.06$ & 1.0 & \\
\hline \multicolumn{7}{|l|}{ Timed barium oesophagram } \\
\hline Oesophagus dilation yes & $275 / 326$ & 87 & $0.27 \pm 0.03$ & $0.33 \pm 0.03$ & 1.0 & 0.36 \\
\hline Oesophagus dilation no & $51 / 326$ & 21 & $0.31 \pm 0.07$ & $0.37 \pm 0.07$ & $1.3(0.8$ to 2.0$)$ & \\
\hline \multicolumn{7}{|l|}{ Manometry before treatment } \\
\hline LOS pressure $\leq 10 \mathrm{~mm} \mathrm{Hg}$ & $10 / 282$ & 3 & $0.29 \pm 0.18$ & $0.29 \pm 0.18$ & 1.0 & 0.91 \\
\hline LOS pressure $>10 \mathrm{~mm} \mathrm{Hg}$ & $272 / 282$ & 88 & $0.25 \pm 0.28$ & $0.32 \pm 0.03$ & $0.9(0.3$ to 3.0$)$ & \\
\hline \multicolumn{7}{|l|}{ Obliteration balloon's waist } \\
\hline Yes & $217 / 327$ & 45 & $0.16 \pm 0.03$ & $0.19 \pm 0.03$ & 1.0 & $<0.001$ \\
\hline No & $110 / 327$ & 62 & $0.48 \pm 0.05$ & $0.60 \pm 0.05$ & 3.6 (2.4 to 5.3$)$ & \\
\hline \multicolumn{7}{|l|}{ Balloon diameter } \\
\hline Consistent $(40 \mathrm{~mm})$ & 212 & 70 & $0.24 \pm 0.03$ & $0.32 \pm 0.04$ & 1.0 & 0.63 \\
\hline $\begin{array}{l}\text { Incremental } \\
(30-35-40 \mathrm{~mm})\end{array}$ & 63 & 16 & $0.27 \pm 0.07$ & $0.37 \pm 0.09$ & $1.1(0.6$ to 1.9$)$ & \\
\hline Other & 61 & 25 & $0.39 \pm 0.06$ & $0.42 \pm 0.07$ & $1.3(0.8$ to 2.0$)$ & \\
\hline \multicolumn{7}{|c|}{ Manometry 3 months after treatment } \\
\hline LOS pressure $\leq 10 \mathrm{~mm} \mathrm{Hg}$ & $82 / 251$ & 15 & $0.17 \pm 0.04$ & $0.19 \pm 0.05$ & 1.0 & 0.03 \\
\hline LOS pressure $>10 \mathrm{~mm} \mathrm{Hg}$ & $169 / 251$ & 61 & $0.25 \pm 0.03$ & $0.33 \pm 0.04$ & $1.9(1.1$ to 3.3$)$ & \\
\hline Total & 336 & 111 & $0.28 \pm 0.03$ & $0.34 \pm 0.03$ & & \\
\hline
\end{tabular}

HR were calculated in univariate Cox regression analysis.

LOS, lower oesophageal sphincter.

\section{Predictors of outcome: univariate analysis}

The risk of recurrence was significantly higher in younger patients, those with classic achalasia, those with incomplete obliteration of the balloon's waist during PD and patients with an LOS pressure greater than $10 \mathrm{~mm} \mathrm{Hg}$ at 3 months after treatment (table 2). The other factors tested, including gender and balloon diameter used during PD, did not influence the risk of recurrence. Furthermore, the presence or absence of oesophageal dilation on a timed barium oesophagram at presentation as well as LOS pressure before treatment did not significantly differ between patients with or without recurrence of achalasia during follow-up.

Table 2 shows that in 10 patients, a presumptive diagnosis of achalasia was made despite low LOS pressures on manometry. In view of the clinical picture with typical symptoms, a fully compatible barium oesophagram, and a non-relaxing LOS with stasis during endoscopy without signs of obstructing lesions, the presumptive diagnosis of achalasia was made and patients were treated with balloon dilation. The response to this therapy in all 10 patients with improvement of both symptoms and barium oesophagram supported the diagnosis of achalasia and suggest that the low LOS pressures were due to misreading in the manometry. These patients were therefore included in our analysis, separate analysis with the exclusion of these 10 patients did not lead to any major changes in results.

\section{Multivariable analysis}

Multivariable analysis showed that age at presentation under 50 years (with increased risk in patients with a younger age), classic achalasia, incomplete obliteration of the balloon's waist during $\mathrm{PD}$ and LOS pressure at 3 months after treatment over $12 \mathrm{~mm} \mathrm{Hg}$ were significant independent factors to predict the development of later recurrence (all $p$ values <0.001). No significant interaction effects were found between these risk factors (all $\mathrm{p}>0.05$ ), and no simple combinations of characteristics of high-risk patients could be found.

\section{Relative hazard threshold in LOS pressure and age}

We examined the relation between LOS pressure measured at 3 months after PD and the risk of recurrence in further detail (figure 2). The higher the LOS pressure post-dilatation, the greater the relative $\mathrm{HR}$ for recurrence. We found a threshold value of $12 \mathrm{~mm} \mathrm{Hg}$. Above this pressure the risk of recurrence increased more or less linearly. Younger age increased the relative HR for recurrence (figure 3). We found a threshold value of 50 years, below which the risk of recurrence increased linearly.

\section{DISCUSSION}

Patients with achalasia are in many countries primarily treated with $\mathrm{PD}$, as this is considered to be a safe and efficient treatment for this condition. Several studies have reported success rates for PD of between $35 \%$ and $85 \% .^{4-11}$ These highly variable results hamper the use of PD as primary treatment for achalasia.

Variation of success rates is possibly due to different periods of follow-up with a gradual decrease in persistent success rates over time. In a study of 72 patients with a mean follow-up of 6.5 years, the risk of recurrence after PD remained as low as $15 \% .{ }^{10}$ Another study showed a persistent treatment success rate of $60 \%$ for a patient population followed for 5-9 years post$\mathrm{PD}$ and $40 \%$ for patients followed more than 15 years post-PD. ${ }^{7}$ 


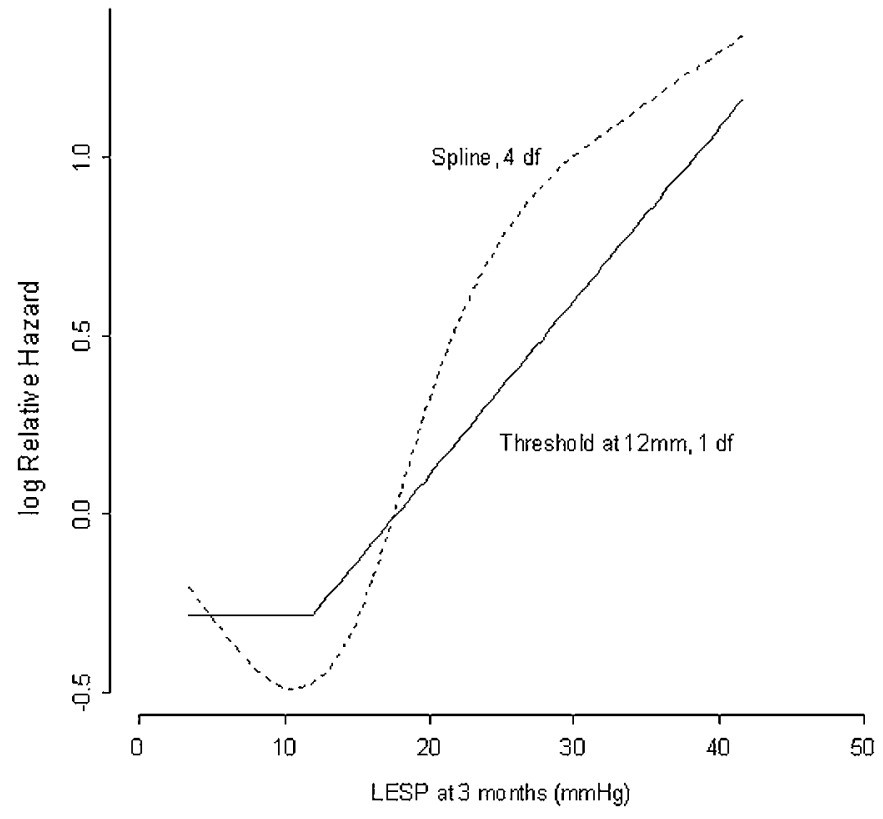

Figure 2 Relative hazard of recurrence of achalasia and lower oesophageal sphincter pressure (LESP) 3 months after pneumatic balloon dilatation. The risk of recurrence increases linearly above a threshold value of $12 \mathrm{~mm} \mathrm{Hg}$.

Our results fit within the above-mentioned range of success. However, given the long follow-up period in our study, we were able to show that the rate of recurrence does indeed increase over time.

Another factor that impairs the direct comparison of different study results is the variable definition of recurrence and success. In some studies, recurrence was defined as symptom recurrence, sometimes based on a symptom score questionnaire such as the Eckardt score, ${ }^{7}$ whereas other studies divided the data on the effectiveness of treatment into four classes according to Vantrappen and Hellemans. ${ }^{18}$ This classification is based on symptoms of dysphagia (duration and frequency), regurgitation

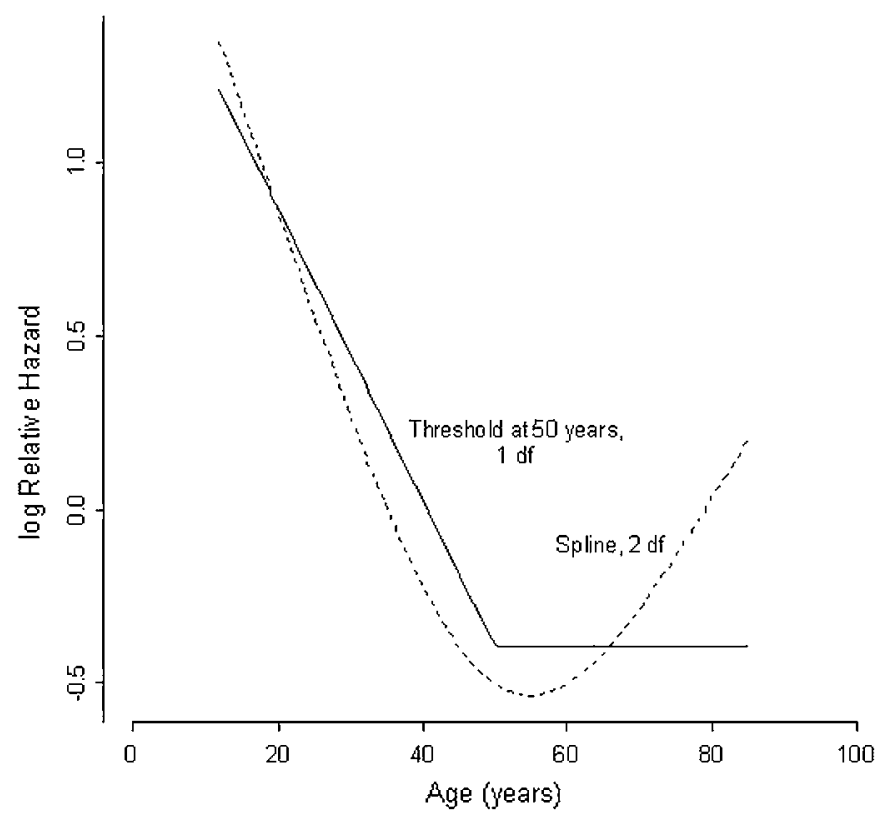

Figure 3 Relative hazard of recurrence of achalasia and age at diagnosis. The risk of recurrence increases linearly below a threshold value of 50 years. and weight loss. Others defined success as the absence of the need for further treatment. ${ }^{10}$ Against this background, we consider our strict protocol of follow-up after PD one of the strengths of our study. In the case of symptom recurrence we performed a gastroscopy, a timed barium oesophagram and a manometry. With this approach, we aimed to confirm an increase in LOS pressures in comparison with previous measurements, thus necessitating repeated treatment.

We deliberately used a clinical definition for persistence and recurrence because this is most applicable and useful in the daily management of achalasia patients. Manometry pressures, timed swallow, or symptoms alone are insufficient tools to diagnose recurrence and repeat $\mathrm{PD}$ or proceed to surgery. Our definition of recurrence was deliberately chosen for optimal clinical management and to prevent misdiagnoses

Another variable factor in the literature concerning PD is the distension protocol used. Until now there has been no strict, uniformly accepted PD protocol for achalasia. Some investigators only perform one dilatation, ${ }^{8}$ others perform several dilatations with incremental diameter of the balloon, or one dilatation at baseline and every couple of weeks after the first dilatation re-dilatation until clinical remission is achieved. ${ }^{5} 719$ In a retrospective study in a group of 75 achalasia patients, graded dilatation was found to be more effective than a single dilatation. ${ }^{20}$ In the present study baseline PD was performed on three consecutive days with balloons of either the same diameter, or incremental (30,35 and $40 \mathrm{~mm}$ ) diameter. According to the distension protocol, the majority of the patients underwent three consecutive dilatations with a balloon diameter of $40 \mathrm{~mm}$ $(n=212)$ or were treated with balloons with increasing diameter, respectively, 30, 35 and eventually $40 \mathrm{~mm}(\mathrm{n}=63)$. There was no significant difference between both groups with respect to the risk of recurrence.

This study shows that 111 out of 336 patients treated with one series of three PD sessions developed recurrence of achalasia requiring further treatment. These first recurrences occurred at a mean interval of 51 months after baseline treatment. The 5 -year risk of recurrence is $28 \%$. In one study with a variable dilation protocol consisting of one to three initial PD sessions, a 5-9 year success of $60 \%$ was found after treatment with PD. In another study, in which the investigators performed one baseline PD followed by on-demand strategy every $2-3$ weeks till clinical remission was achieved, a 5 -year persistent success of $67 \%$ was reported. ${ }^{5}$ These studies are in contrast to a study from German investigators, which reported no more than $40 \%$ persistent remission 5 years after one baseline $\mathrm{PD}{ }^{8}$ In comparison with those studies, our 5 -year risk of recurrence is relatively low.

After $\mathrm{PD}$, all patients in the present study had a strict schedule of follow-up at 3, 12, 24 months and then every 2 years. Surveillance included gastroscopy, manometry and timed barium oesophagram. After 8 years of follow-up surveillance including gastroscopy, further examination was performed on indication. In another study, patients were not routinely followed up after $\mathrm{PD}$, but were advised to make an appointment when symptoms recurred. ${ }^{7}$ We believe that symptoms alone are not reliable in assessing treatment success, but an objective assessment of treatment and symptoms is required to achieve a higher treatment success. Patients are used to having a certain level of discomfort due to achalasia. Therefore this group of patients will not present themselves at an early phase of recurrence of disease.

In several studies risk factors for symptom recurrence after PD have been identified. These factors were mainly young age (<40 years), a post-treatment LOS pressure above $10 \mathrm{~mm} \mathrm{Hg}$ and a short period of symptoms before treatment. ${ }^{5} \quad \begin{array}{lll} & 12\end{array}$ 
A severely dilated oesophagus ( $>80 \mathrm{~mm}$ ) has been reported to be associated with poor outcome. ${ }^{49}$

We found age to be a predictive factor in treatment outcome. Patients younger than 50 years responded less well to pneumatic dilatation. The risk of recurrence increased linearly in patients with an age below 50 years. High recurrence percentages were found in patients younger than 21 years as the 5 -year and 10 -year risks of recurrence were $64 \%$ and $72 \%$, respectively.

In one study achalasia was categorised into three subtypes; type I as classic achalasia, type II as achalasia with oesophageal compression and type III as achalasia with spastic contractions. That study analysed treatment outcome in 83 patients and showed each subtype to be distinct in their responsiveness to therapy. Type III was a predictor of negative treatment response. ${ }^{21}$ The current study differentiated achalasia into two subtypes, classic achalasia (type I) and vigorous achalasia (type II). This differentiation could be made in 288 patients. We showed that classic achalasia was associated with a higher risk of recurrence.

In several studies treatment response was associated with low LOS pressures post-dilatation. The most optimal response was found in patients with a post-dilatation LOS pressure lower than $10 \mathrm{~mm} \mathrm{Hg}{ }^{8}{ }^{11} 14$ The current study confirms that a low LOS pressure post-dilation is associated with a good clinical outcome and shows that a LOS pressure of $12 \mathrm{~mm} \mathrm{Hg}$ or less at 3 months post-dilatation is associated with a significantly lower risk of recurrence. We therefore recommend the performance of manometry at 3 months post-dilatation to measure LOS pressure as a predictor of treatment outcome.

Incomplete obliteration of the balloon's waist was found to be a significant predictor of clinical outcome as this subgroup showed a significantly higher risk of the development of recurrence. In comparison with patients with complete obliteration, patients without showed a HR of 3.6.

In the present study the presence of dilation of the oesophagus was not associated with an increased risk of recurrence; however, we only scored the parameter dilation/no dilation but did not grade the severity of oesophageal dilation. This does not exclude the possibility that the level of severity of dilation could correlate with the risk of recurrence.

In a study from the USA, investigators showed that patients with a longer duration of symptoms before diagnosis had a more tortuous oesophageal body and tended to have an increased oesophageal diameter observed by radiography. ${ }^{22}$ It can be hypothesised that early detection of recurrent disease and repeated treatment at an early stage may prevent (further) oesophageal decompensation. Further investigation needs to be performed to confirm this hypothesis.

There are a few shortcomings to our study. First, we did not correlate improved emptying on a barium oesophagram independently with symptom relief, LOS pressures and relapse rates. Second, our pneumatic dilatations stopped at a pressure of $300 \mathrm{~mm} \mathrm{Hg}$. We did not increase the balloon pressure until complete obliteration of the balloon waist was achieved in all patients. Therefore our results are not the ultimate standard that can be achieved by PD treatment. Further studies have to show whether differentiation of the approach, for example higher pressure, can improve results without increasing complication rates.

Several studies have compared surgery and pneumatic dilatation for the treatment of patients with achalasia. ${ }^{19}{ }^{23-26}$

In one of these studies $30.3 \%$ of patients initially treated with surgical myotomy had another intervention after 5 years and $37.5 \%$ of patients had a subsequent intervention 10 years after initial therapy. ${ }^{26}$ In our study we obtained similar results with
$\mathrm{PD}$, with a 5 and 10 -year recurrence rate of $28 \%$ and $34 \%$, respectively.

A recently published systemic review showed that laparoscopic myotomy combined with an antireflux procedure provided better symptom relief $(90 \%)$ than all endoscopic and other surgical approaches, with a low complication rate (6.3\%). ${ }^{27}$

Recently, a large European prospective randomised study compared laparoscopic Heller myotomy versus PD as baseline treatment in 200 achalasia patients. ${ }^{28}$ One hundred and six patients were treated with laparoscopic Heller myotomy with Dor fundoplication and 94 patients were treated with pneumatic dilation. After 2 years of follow-up both treatments had a comparable success rate of $92 \%$ versus $87 \%$. Further follow-up is required to evaluate long-term outcome. The long-term outcome of this study should further determine which therapy should be reserved for different subgroups of achalasia patients as baseline therapy.

In summary, we conclude that $\mathrm{PD}$ is an effective treatment for achalasia, but that recurrence rates after PD increase over time. Young age at presentation, classic achalasia, incomplete obliteration of the balloon's waist during PD, and high LOS pressure at 3 months after $\mathrm{PD}$ are independent predicting factors of the need for repeated PD during follow-up. These factors should be taken into account in the follow-up strategy of achalasia patients, leading to a stricter follow-up protocol in patients with the above-mentioned predicting factors. Such a protocol should aim for the timely detection of recurrence (defined as symptom recurrence in combination with objective parameters such as a significant increase in LOS pressure) with renewed, tailored treatment. Our results show that most patients are then eligible for renewed PD. However, selected cases such as those 21 years of age or younger may in the case of recurrence be referred for surgery earlier.

\section{Competing interests None.}

Provenance and peer review Not commissioned; externally peer reviewed.

\section{REFERENCES}

1. Richter JE. Oesophageal motility disorders. Lancet 2001;358:823-8.

2. Park W, Vaezi MF. Etiology and pathogenesis of achalasia: the current understanding. Am J Gastroenterol 2005;100:1404-14.

3. Lake JM, Wong RK. Review article: the management of achalasia - a comparison of different treatment modalities. Aliment Pharmacol Ther 2006;24:909-18.

4. Karamanolis G, Sgouros S, Karatzias G, et al. Long-term outcome of pneumatic dilation in the treatment of achalasia. Am J Gastroenterol 2005;100:270-4.

5. Zerbib F, Thetiot V, Richy F, et al. Repeated pneumatic dilations as long-term maintenance therapy for esophageal achalasia. Am J Gastroenterol 2006;101:692-7.

6. Parkman HP, Reynolds JC, Ouyang A, et al. Pneumatic dilatation or esophagomyotomy treatment for idiopathic achalasia: clinical outcomes and cost analysis. Dig Diseases Sci 1993;38:75-85.

7. West RL, Hirsch DP, Bartelsman JF, et al. Long term results of pneumatic dilation in achalasia followed for more than 5 years. American $\mathrm{J}$ Gastroenterol 2002; 97:1346-51.

8. Eckardt VF, Gockel I, Bernhard G. Pneumatic dilation for achalasia: late results of a prospective follow up investigation. Gut 2004;53:629-33.

9. Vantrappen G, Hellemans J, Deloof W, et al. Treatment of achalasia with pneumatic dilatations. Gut 1971;12:268-75

10. Katz PO, Gilbert J, Castell DO. Pneumatic dilatation is effective long-term treatment for achalasia. Dig Dis Sci 1998;43:1973-7.

11. Eckardt VF, Aignherr C, Bernhard G. Predictors of outcome in patients with achalasia treated by pneumatic dilation. Gastroenterology 1992;103:1732-8

12. Fellows IW, Ogilvie AL, Atkinson M. Pneumatic dilatation in achalasia. Gut 1983;24:1020-3.

13. Azizkhan RG, Tapper D, Eraklis A. Achalasia in childhood: a 20-year experience. J Pediatr Surg 1980;15:452-6

14. Ponce J, Garrigues V, Pertejo V, et al. Individual prediction of response to pneumatic dilation in patients with achalasia. Dig Dis Sci 1996;41:2135-41.

15. Vaezi MF, Baker ME, Achkar E, et al. Timed barium oesophagram: better predictor of long term success after pneumatic dilation in achalasia than symptom assessment. Gut 2002;50:765-70. 
16. Harrell FE. Regression modeling strategies. With applications to linear models, logistic regression, and survival analysis. New York: Springer Series in Statistics, 2001.

17. Steyerberg E. Clinical prediction models: a practical approach to development, validation, and updating. New York: Springer, 2009.

18. Vantrappen G, Hellemans J. Treatment of achalasia and related motor disorders Gastroenterology 1980:79:144-54.

19. Vela MF, Richter JE, Khandwala F, et al. The long-term efficacy of pneumatic dilatation and Heller myotomy for the treatment of achalasia. Clin Gastroenterol Hepatol 2006:4:580-7.

20. Farhoomand K, Connor JT, Richter JE, et al. Predictors of outcome of pneumatic dilation in achalasia. Clin Gastroenterol Hepatol 2004;2:389-94.

21. Pandolfino JE, Kwiatek MA, Nealis T, et al. Achalasia: a new clinically relevant classification by high-resolution manometry. Gastroenterology. 2008; 135:1526-33.

22. Shiino Y, Houghton SG, Filipi CJ, et al. Manometric and radiographic verification of esophageal body decompensation for patients with achalasia. J Am Coll Surg 1999:189:158-63.

\section{Editor's quiz: GI snapshot}

\section{Gastric polypoid lesion after gastrectomy}

\section{CLINICAL PRESENTATION}

An 87-year-old man presented for endoscopy for investigation of anorexia, abdominal bloating and fullness for the previous 2 months. He had had a subtotal gastrectomy with a Billroth II anastomosis 40 years previously for a gastric ulcer with bleeding. Upper endoscopy revealed a $20 \mathrm{~mm}$ diameter, broad-based polypoid lesion with a smooth surface at the greater curvature of the middle body of the stomach (figure 1). Biopsies from the surface mucosa showed mild chronic inflammation and mild foveolar hyperplasia and were negative for Helicobacter pylori infection. Abdominal CT revealed a $2 \times 2 \mathrm{~cm}$ protruding smooth nodular lesion in an area in keeping with a submucosal lesion such as a gastrointestinal stromal tumour (figure 2). Endoscopic ultrasonography showed a hypoechoic homogenous lesion within the secondary echo layer of the stomach. Based on the image findings, the lesion was diagnosed as a gastrointestinal stromal tumour.

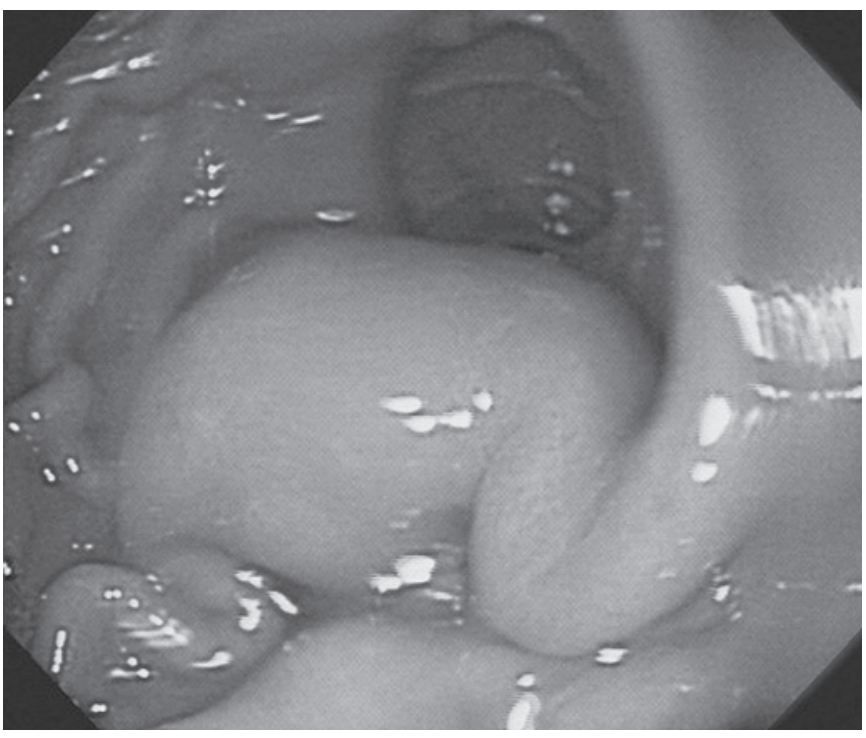

Figure 1 Upper endoscopy revealed a $20 \mathrm{~mm}$ diameter, broad-based polypoid lesion with a smooth surface at the greater curvature side of middle body of stomach.
23. Csendes A, Braghetto I, Henriquez A, et al. Late results of a prospective randomised study comparing forceful dilatation and oesophagomyotomy in patients with achalasia. Gut 1989;30:299-304.

24. Csendes A, Velasco N, Braghetto I, et al. A prospective randomized study comparing forceful dilatation and esophagomyotomy in patients with achalasia of the esophagus. Gastroenterology 1981:80:789-95

25. Gockel I, Junginger T, Eckardt VF. Effects of pneumatic dilation and myotomy on esophageal function and morphology in patients with achalasia. American Surg 2005:71:128-31.

26. Lopushinsky SR, Urbach DR. Pneumatic dilatation and surgical myotomy for achalasia. JAMA 2006;296:2227-33.

27. Campos GM, Vittinghoff E, Rabl C, et al. Endoscopic and surgical treatments for achalasia: a systematic review and meta-analysis. Ann Surgery 2009;249:45-57.

28. Boeckxstaens GE, Annese V, des Varannes SB, et al. The European Achalasia Trial: a randomized multi-centre trial comparing endoscopic pneumodilation and laparoscopic myotomy as primary treatment of idiopathic achalasia. Gastroenterology 2010;138(Suppl 1):S53.

\section{QUESTION}

What is the differential diagnosis of the endoscopic image? See page 48 for the answer

\section{Huan-Lin Chen, ${ }^{1}$ Ming-Jong Bair, ${ }^{1}$ I-Tsung Lin, ${ }^{1}$ Chia-Hsien Wu, Yu-Jan Chen ${ }^{2}$}

'Division of Gastroenterology, Department of Internal Medicine, Mackay Memorial Hospital, Mackay Medicine, Taitung-Branch, Taiwan; '2Division of Pathology, Department of Internal Medicine, Mackay Memorial Hospital, Taipei, Taiwan

Correspondence to Dr Ming-Jong Bair, Division of Gastroenterology, Department of Internal Medicine, Mackay Memorial Hospital, Taitung Branch, No. 1, Lane 303 Changsha Street, Taitung, Taiwan; a5963@ttms.mmh.org.tw

Competing interests None.

Patient consent Obtained.

Provenance and peer review Not commissioned; externally peer reviewed. 